\title{
Impact of heart failure on stroke mortality and recurrence
}

\author{
Tiberiu A Pana, ${ }^{1}$ Adrian D Wood, ${ }^{1}$ Jesus A Perdomo-Lampignano, ${ }^{1}$ \\ Somsak Tiamkao, ${ }^{2,3}$ Allan B Clark, ${ }^{4}$ Kannikar Kongbunkiat, ${ }^{2,5}$ \\ Joao H Bettencourt-Silva, ${ }^{6}$ Kittisak Sawanyawisuth, ${ }^{5}$ Narongrit Kasemsap, ${ }^{3,5}$ \\ Mamas A Mamas, ${ }^{7}$ Phyo K Myint ${ }^{1}$
}

\begin{abstract}
- Additional material is published online only. To view please visit the journal online (http://dx.doi.org/10.1136/ heartasia-2018-011139).

${ }^{1}$ Ageing Clinical and Experimental Research Team, Institute of Applied Health

Sciences, School of Medicine, Medical Sciences and Nutrition, University of Aberdeen, Aberdeen, UK

${ }^{2}$ Neurology Division, Department of Medicine, Faculty of Medicine, Khon Kaen University, Khon Kaen, Thailand

${ }^{3}$ North-Eastern Stroke Research Group, Khon Kaen University, Khon Kaen, Thailand

${ }^{4}$ Norwich Medical School, University of East Anglia, Norwich, UK

${ }^{5}$ Ambulatory Medicine Division, Department of Meidicine, Faculty of Medicine, Khon Kaen University, Khon Kaen, Thailand ${ }^{6}$ Clinical informatics, Department of Medicine, University of Cambridge, Cambridge, UK ${ }^{7}$ Keele Cardiovascular Research Group, Centre for Prognosis Research, Institute of Primary Care and Health Sciences, Keele University, Stoke-on-Trent, UK
\end{abstract}

\section{Correspondence to} Professor Phyo K Myint, Institute of Applied Health Sciences, University of Aberdeen, Aberdeen AB25 2ZD, UK; phyo. myint@abdn.ac.uk

Received 2 November 2018

Revised 1 March 2019

Accepted 6 March 2019

\section{Check for updates}

(C) Author(s) (or their employer(s)) 2019. No commercial re-use. See rights and permissions. Published by BMJ.

\begin{tabular}{l}
\hline To cite: Pana TA, \\
Wood AD, Perdomo- \\
Lampignano JA, et al. Heart \\
Asia 2019;11:e011139. \\
doi:10.1136/ \\
heartasia-2018-011139
\end{tabular}

\section{ABSTRACT}

Objective We aimed to examine the impact of heart failure (HF) on stroke mortality (in-hospital and postdischarge) and recurrence in a national stroke cohort from Thailand.

Methods We used a large, insurance-based database including all stroke admissions in the public health sector in Thailand between 2004 and 2015. Logistic and Royston-Parmar regressions were used to quantify the effect of HF on in-hospital and long-term outcomes, respectively. All models were adjusted for age, sex and comorbidities and stratified by stroke type: acute ischaemic stroke (AIS) or intracerebral haemorrhage (ICH). Multistate models were constructed using flexible survival techniques to predict the impact of HF on the disease course of a patient with stroke (baseline[recurrence]-death). Only first-ever cases of AIS or ICH were included in the multistate analysis.

Results 608890 patients (mean age $64.29 \pm 13.72$ years, $55.07 \%$ men) were hospitalised (370 527 AIS, $173236 \mathrm{ICH}$ and 65127 undetermined pathology). There were 398663 patients with first-ever AIS and ICH. Patients were followed up for a median $(95 \% \mathrm{Cl})$ of 4.47 years (4.45 to 4.49). HF was associated with an increase in postdischarge mortality in AIS $(\mathrm{HR}[99 \% \mathrm{Cl}] 1.69[1.64$ to 1.74$]$ ) and ICH (2.59 [2.07 to 3.26]). HF was not associated with AIS recurrence, while ICH recurrence was only significantly increased within the first 3 years after discharge (1.79 [1.18 to 2.73]).

Conclusions $\mathrm{HF}$ increases the risk of mortality in both AIS and ICH. We are the first to report on high-risk periods of stroke recurrence in patients with HF with $\mathrm{ICH}$. Specific targeted risk reduction strategies may have significant clinical impact for mortality and recurrence in stroke.

\section{INTRODUCTION}

Heart failure (HF) is a recognised risk factor for stroke incidence. The proposed mechanisms underlying the association between HF and increased risk of acute ischaemic stroke (AIS) include thromboembolism, cerebral hypoperfusion and small vessel endothelial damage. ${ }^{1}$ The association between $\mathrm{HF}$ and intracerebral haemorrhage (ICH) is less clearly understood, but may relate to antithrombotic therapy being administered in patients with $\mathrm{HF}$, particularly in those with ischaemic cardiomyopathy or atrial fibrillation (AF). ${ }^{2}$

Given the increased stroke incidence in patients with HF, hypotheses regarding the influence of pre-existing HF on acute stroke outcomes have

\section{Key messages}

What is already known about this subject?

- Heart failure is associated with higher mortality and recurrence rates after ischaemic stroke.

- Previous literature results are based on Western populations.

What does this study add?

- This is the first study to examine this relationship in an Asian stroke population.

- Heart failure predicts higher mortality outcomes in patients with ischaemic stroke and in patients with haemorrhagic stroke.

- Heart failure is associated with higher recurrence rates in the medium-term follow-up for haemorrhagic strokes only.

How might this impact on clinical practice?

- Clinicians should be aware that patients with haemorrhagic stroke with heart failure are at higher risk of both mortality and recurrence in the medium-term after discharge (up to 3 years).

- This study should be replicated in other Asian populations.

been formulated. ${ }^{3}$ Previous studies have mainly analysed the association between prevalent HF and post-AIS outcomes, suggesting that it may be associated with higher mortality, longer in-hospital stay, and higher disability and recurrence rates. ${ }^{14-8}$ Nevertheless, there have been no studies evaluating the impact of HF on the outcomes of ICH.

In the current study, we sought to determine the impact of HF on in-hospital and long-term outcomes (mortality and stroke-specific recurrence) in both major stroke subtypes (AIS and ICH) using a large unselected cohort of patients with stroke. Identification of $\mathrm{HF}$ as a risk factor for stroke mortality and recurrence may drive further research for the identification and testing of targeted interventions in this population.

\section{METHODS}

Data acquisition, case ascertainment and variable coding

We used a large observational cohort of consecutively admitted patients with stroke from Thailand, drawn from the Universal Coverage Health 
Insurance Database. Therefore, the cohort consists of patients admitted to hospitals with a primary diagnosis of stroke. Details regarding the health insurance systems in Thailand can be found in our previous reports. ${ }^{9}{ }^{10}$ Eligible patients were selected based on the primary diagnosis ICD-10 (International Classification of Diseases, 10th revision) code recorded in the database. All patients covered by the basic health insurance system ever admitted between October 2004 and September 2015 with a diagnosis of stroke (ICD-10 codes I61 [intracerebral haemorrhage], I63 [cerebral infarction] and I64 [stroke of undetermined pathology]) were included in this study. Pre-existing comorbidity, demographic and clinical data were extracted from insurance reimbursement forms using ICD-10 codes, detailed in online supplementary table 1 .

Further admissions with a primary diagnosis of stroke or HF (ICD-10) and the vital status (date of death if applicable) were recorded. A recurrent stroke event during the follow-up was extracted only if the primary diagnosis of this admission matched the one of the index stroke. In order to account for any new HF diagnoses, we also assessed subsequent hospital admissions, for any changes in the HF comorbidity status.

\section{Inclusion criteria}

Inclusion criteria, outcomes of interest (mortality and stroke recurrence) and selection of study variables were all determined a priori. A total of 615758 of patients with stroke were admitted with a primary diagnosis of stroke in the database. After excluding patients younger than $18(\mathrm{n}=1427)$ or older than 100 $(\mathrm{n}=43)$ and those with a missing postdischarge data $(\mathrm{n}=5419)$, a total of 608890 patients with acute stroke were included.

For the outcome of stroke recurrence, a further 81530 patients with prevalent stroke (ICD-10 I61, I63 and I64) were excluded, as well as 128697 patients with stroke who were diagnosed with a stroke of undetermined pathology on their index admission, yielding a cohort of 398663 patients with first-ever AIS and ICH.

\section{Statistical analysis}

All analyses were performed using Stata V.14.2 SE, Stata Statistical Software. Due to the large sample size, we chose a $\mathrm{p}$ value $<0.01$ to represent the threshold of statistical significance.

\section{Descriptive statistics}

Stroke type-specific descriptive characteristics were compared between those with prevalent HF and those without at baseline (index admission in the database) using the independent-sample $\mathrm{t}$-test, Mann-Whitney $\mathrm{U}$ test and $\chi^{2}$ test, as appropriate.

\section{Analysis of in-hospital mortality}

Logistic regressions were used to examine the impact of HF on in-hospital mortality. We performed analyses for the entire cohort and then stratified by three categories (ischaemic, haemorrhagic and stroke of undetermined pathology). In order to minimise confounding, we selected covariates according to previous literature $^{4-8}$ to be included in the multivariable logistic regression model. All models were adjusted for age, sex, comorbidities (hypertension, diabetes mellitus, dyslipidaemia, ischaemic heart disease, valvular disease, atrial fibrillation, other arrhythmias, peripheral vascular disease, cancers, chronic obstructive pulmonary disease, liver disease, anaemia, alcohol-related disorders and neurodegenerative disorders) and admission parameters (fever, hypoglycaemia and hyperglycaemia). Additional sensitivity analyses were performed with adjustments limited to age, sex, cancer and important cardiovascular comorbidities (hypertension,

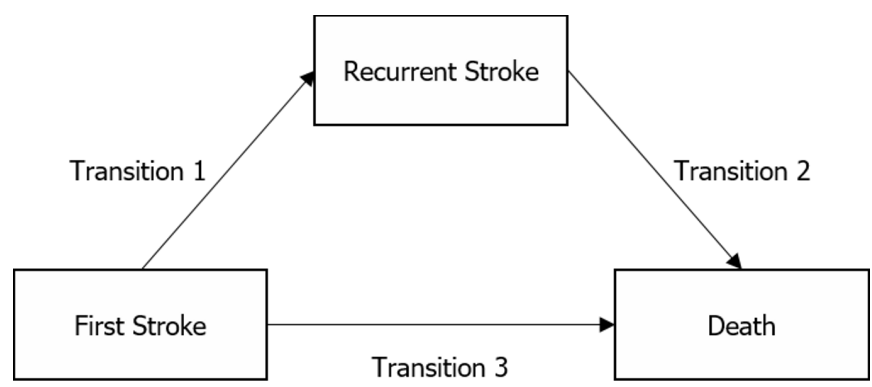

Figure 1 Transitions used in creating the multistate models.

diabetes, hyperlipidaemia, ischaemic heart disease, valvular heart disease, atrial fibrillation, other arrhythmias and peripheral vascular disease).

\section{Analysis of long-term postdischarge mortality}

To examine the influence of HF on long-term mortality, we constructed a survival model based on a flexible parametric regression, ${ }^{11}$ using restricted cubic splines (RCS). The number of internal knots was chosen pragmatically in line with existing literature ${ }^{1213}$ for our sample size. The proportional hazards $(\mathrm{PH})$ assumption was verified using log-negative-log plots. The HRs for the variables which violated the $\mathrm{PH}$ assumption were modelled parametrically using the Weibull distribution. When HF was found to violate the $\mathrm{PH}$ assumption, the $\mathrm{HR}$ was modelled using RCS with five internal knots. Models were adjusted for the same confounders as above. Any new HF diagnosis after discharge was included as a binary time-updated variable in the models.

\section{Analysis of long-term postdischarge mortality and recurrence}

We constructed three transition multistate models illustrating the typical natural course of a patient with stroke (figure 1). This part of the analysis was confined to first-ever stroke cases, given that one of the outcomes of the multistate model was stroke recurrence. Furthermore, only stroke cases with an identified stroke type (either AIS or ICH) were included. Each transition was modelled as a flexible parametric regression using RCS. The $\mathrm{PH}$ assumption was verified using log-negative-log plots for the exposure variable. Where this assumption was found to be violated, the exposure variable was introduced in the models as a function of RCS with five internal knots. Any new diagnoses of HF or any adjusting comorbidities were updated into the models, taking into account the number of days postdischarge when they occurred. Additional sensitivity analyses were performed with adjustments limited to age, sex, cancer and important cardiovascular comorbidities (hypertension, diabetes, hyperlipidaemia, ischaemic heart disease, valvular heart disease, atrial fibrillation, other arrhythmias and peripheral vascular disease).

\section{RESULTS}

\section{Patient population and descriptive statistics}

A flow chart of the patient population is illustrated in figure 2 . After applying the exclusion criteria, a total of 608890 stroke cases were included. There were 370527 (60.85\%) cases of AIS, $173236(28.45 \%)$ cases of ICH and $65127(10.70 \%)$ cases of stroke with an undetermined pathology.

Patient characteristics on admission are summarised in table 1. Patients had a mean age (SD) of 64.3 (13.7) years, and 55.1\% were male. Patients with pre-existing HF (compared with those without) were older, more likely to be female, spend more time in hospital and have a higher comorbidity burden across 


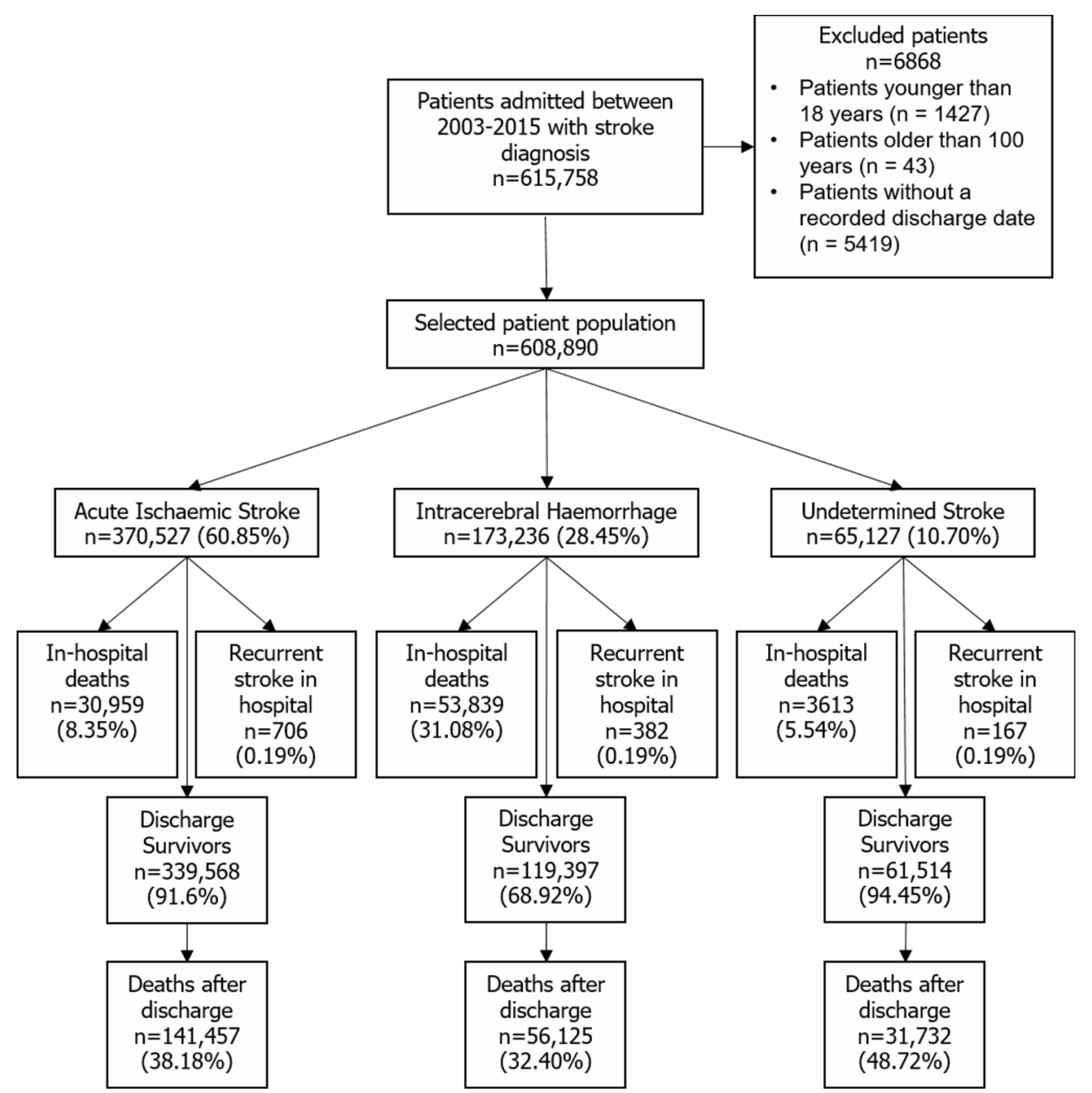

Figure 2 Patient population flow chart.

all stroke types. The admission parameters, in-hospital complications and procedures are summarised table 2. Patients with pre-existing HF (compared with those without) were more likely to be hypoglycaemic on admission, die in hospital and suffer complications (respiratory failure, pneumonia, sepsis, shock, myocardial infarction, acute kidney injury, pulmonary oedema) during the hospital stay across all stroke types. Patients with HF who were admitted with AIS were more likely to be comatose in-hospital. Patients with HF and AIS were more likely to receive intravenous thrombolytic treatment than those without.

\section{Analysis of in-hospital mortality}

The results of the multivariable logistic regressions with in-hospital death as the outcome are illustrated in table 3. Pre-existing HF was associated with increased odds of in-hospital death in all four models. Overall, there was an increase in odds, while the models fitted separately for each stroke type showed the biggest effect in AIS followed by strokes of undetermined pathology and $\mathrm{ICH}$. The sensitivity analysis with adjustments confined to cardiovascular comorbidities and cancer (online supplementary table 2) showed a higher effect for all stroke types, although not significantly different from the main model. For the sensitivity analysis, OR (99\% CI) for all strokes, AIS, ICH and strokes of undetermined pathology were 1.86 (1.78 to 1.93$), 2.46$ (2.34 to $2.58), 1.47$ (1.35 to 1.60$)$ and 1.92 (1.62 to 2.28$)$, respectively.

\section{Analysis of long-term postdischarge mortality}

All patients were followed up for a maximum 4108 days for this outcome. Patients with ischaemic, haemorrhagic and undetermined strokes were followed up for a median (95\% CI) of 1518 (1511 to 1525$), 1731$ (1715 to 1745) and 2409 (2385 to 2431 ) days, respectively. Online supplementary table 3 details the predictors which were included in each model and those that were introduced as time-varying covariates. Comorbid HF was significantly associated with an excess mortality risk in all the stroke categories, as well as overall. For strokes of undetermined pathology, the HR (99\% CI) was 2.01 (1.88 to 2.15), while for AIS the HR was 1.97 (1.92 to 2.03). Because the HR was calculated for patients with $\mathrm{ICH}$ using RCS, the resulting function was not constant with time, and is illustrated in online supplementary figurer 1 . This estimate is significant throughout most of the follow-up period. The HR becomes significant on the 13th day postdischarge HR $(t=13)=1.31$ (1.04 to 1.64$)$, after which it increases to a maximum at the end of the follow-up period HR $(t=4108)=2.56(2.03$ to 3.22$)$.

Online supplementary figure 2 and online supplementary table 4 illustrate the predicted survival functions stratified by HF status and stroke type. Overall, the survival rate is about half for those with HF who survived the index stroke compared with their counterparts without HF.

Analysis of long-term postdischarge mortality and recurrence For both mortality and recurrence, all patients were followed up for a maximum of 4108 days. For the mortality outcome, patients with ischaemic and haemorrhagic strokes were followed up for a median (95\% CI) of 1583 (1577 to 1590) and 1802 (1792 to 1814) days, respectively. For the recurrent stroke outcome, patients with ischaemic and haemorrhagic strokes were followed 
Table 1 Patient characteristics and comorbid conditions at hospital admission

\begin{tabular}{|c|c|c|c|c|c|c|c|c|}
\hline & \multicolumn{2}{|l|}{ All strokes } & \multicolumn{2}{|c|}{ Acute ischaemic stroke } & \multicolumn{2}{|c|}{ Intracerebral haemorrhage } & \multicolumn{2}{|c|}{$\begin{array}{l}\text { Stroke of undetermined } \\
\text { pathology }\end{array}$} \\
\hline & HF $(n=15397)$ & $\begin{array}{l}\text { No HF }(n=593 \\
\text { 493) }\end{array}$ & HF $(n=11522)$ & $\begin{array}{l}\text { No HF }(n=359 \\
\text { 005) }\end{array}$ & HF $(n=2464)$ & $\begin{array}{l}\text { No HF }(n=170 \\
772)\end{array}$ & $H F(n=1411)$ & $\begin{array}{l}\text { No HF }(n=63 \\
716)\end{array}$ \\
\hline Age & $66.97(13.81)$ & $64.22(13.71)$ & $67.49(13.70)$ & $65.47(13.24)$ & $63.69(14.08)$ & $61.12(14.28)$ & $68.46(1.40)$ & $65.47(13.41)$ \\
\hline \multicolumn{9}{|l|}{ Sex } \\
\hline Male & $6989(45.40)$ & $328328(55.32)$ & $5174(44.91)$ & $192553(53.64)$ & $1204(48.86)$ & $102204(59.85)$ & $611(43.30)$ & $33571(52.69)$ \\
\hline Female & $8408(54.61)$ & 265165 (44.68) & $6348(55.09)$ & 166452 (46.36) & $1260(51.14)$ & $68568(40.15)$ & $800(56.70)$ & $30145(47.31)$ \\
\hline Length of stay & $6(3-12)$ & $3(2-7)$ & $6(3-12)$ & $3(2-6)$ & $7(3-19)$ & $5(2-11)$ & $4(2-8)$ & $3(2-5)$ \\
\hline \multicolumn{9}{|l|}{ Comorbidities on admission } \\
\hline Hypertension & $8398(54.54)$ & 289539 (48.79) & $6037(52.40)$ & 170207 (47.41) & $1660(67.37)$ & $93184(54.57)$ & $701(49.68)$ & $26148(41.04)$ \\
\hline Diabetes mellitus & $3565(23.15)$ & 100563 (16.94) & $2729(23.69)$ & 72705 (20.25) & $538(21.83)$ & $16379(9.59)$ & $298(21.12)$ & $11479(18.02)$ \\
\hline Dyslipidaemia & $3859(25.06)$ & $139704(23.54)$ & $3180(27.60)$ & $112032(31.21)$ & $390(15.83)$ & $15097(8.84)$ & $289(20.48)^{*}$ & $12575(19.74)^{*}$ \\
\hline Ischaemic heart disease & $2400(15.59)$ & $16161(2.72)$ & $1861(16.15)$ & $12196(3.40)$ & $289(11.73)$ & $2251(1.32)$ & $250(17.72)$ & $1714(2.69)$ \\
\hline Valvular disease & $1626(10.56)$ & $8746(1.47)$ & $1326(11.51)$ & $7022(1.96)$ & $174(7.06)$ & $862(0.50)$ & $126(8.93)$ & $862(1.35)$ \\
\hline Atrial fibrillation & $5075(32.96)$ & $35173(5.93)$ & $4230(36.71)$ & $29240(8.14)$ & $453(18.38)$ & $2973(1.74)$ & $392(27.78)$ & $2960(4.65)$ \\
\hline Arrhythmia & $421(2.73)$ & $4374(0.74)$ & $331(2.87)$ & $3259(0.91)$ & $55(2.23)$ & $657(0.38)$ & $35(2.48)$ & $458(0.72)$ \\
\hline Peripheral vascular disease & $108(0.70)$ & $1486(0.25)$ & $85(0.74)$ & $1067(0.30)$ & $16(0.65)$ & $316(0.19)$ & $7(0.50)$ & $103(0.16)$ \\
\hline Cancers & $103(0.67)^{*}$ & $3136(0.53)^{*}$ & $89(0.77)^{*}$ & $2094(0.58)^{*}$ & $10(0.41)^{*}$ & $753(0.44)^{*}$ & $4(0.28)^{*}$ & $289(0.45)^{*}$ \\
\hline $\begin{array}{l}\text { Chronic obstructive } \\
\text { pulmonary disease }\end{array}$ & $756(4.91)$ & $10131(1.71)$ & $563(4.89)$ & $6856(1.91)$ & $107(4.34)$ & $2126(1.24)$ & $86(6.09)$ & $1149(1.80)$ \\
\hline Chronic kidney disease & $2085(13.54)$ & $31173(5.25)$ & $1549(13.44)$ & $21167(5.90)$ & $355(14.41)$ & $6850(4.01)$ & $181(12.83)$ & 3156 (4.95) \\
\hline Liver disease & $307(1.99)$ & $7302(1.23)$ & $222(1.93)$ & $3597(1.00)$ & $62(2.52)^{*}$ & $3149(1.84)^{*}$ & $23(1.63)$ & $556(0.87)$ \\
\hline Anaemia & $2190(14.22)$ & 39809 (6.71) & $1547(13.43)$ & $22748(6.34)$ & $476(19.32)$ & $14071(8.24)$ & $167(11.84)$ & $2990(4.69)$ \\
\hline Alcohol-related disorders & $92(0.60)$ & $7129(1.20)$ & $66(0.57)$ & $3151(0.88)$ & $22(0.89)$ & $3485(2.04)$ & $4(0.28)^{*}$ & $493(0.77)^{*}$ \\
\hline $\begin{array}{l}\text { Neurodegenerative } \\
\text { disorders }\end{array}$ & $251(1.63)$ & $5811(0.98)$ & $213(1.85)$ & $4695(1.31)$ & $18(0.73)$ & $607(0.36)$ & $20(1.42)^{*}$ & $509(0.80)^{*}$ \\
\hline
\end{tabular}

Continuous normally distributed variables (age) are presented as mean (SD), while continuous non-normal variables (length of stay) are presented as median (IQR). Categorical variables are presented as frequency (percentage). All between-group differences are statistically significant $(p<0.01)$ unless otherwise specified.

*Between-group difference not statistically significant $(p>0.01)$.

$\mathrm{HF}$, heart failure.

up for a median (95\% CI) of 875 (862 to 887 ) and 778 (767 to 789 ), respectively. The median follow-up times and $95 \%$ CIs were calculated using the reverse Kaplan-Meier method. online supplementary figure 3 illustrates the first-ever stroke patient population, stratified by stroke type. A total of 302603 patients with first AIS survived to hospital discharge without a recurrent event. Post-discharge recurrent AIS occurred in 32036 patients (10.59\%). A total of $128850(42.58 \%)$ patients died during follow-up, 112073 (37.04\%) after their first-ever stroke and 16777 (5.54\%) after their recurrent AIS. We recorded 96060 patients with first-ever ICH who survived to hospital discharge without having experienced stroke recurrence. Post-discharge recurrent ICH occurred in $7548(7.86 \%)$ patients. A total of 45 335 (47.19\%) patients died during follow-up, 40609 (42.27\%) after their first-ever ICH and 4726 (4.92\%) after their recurrent haemorrhage.

Online supplementary table 5 and figure 3 illustrate the HRs for each transition for each stroke type, calculated for patients with comorbid HF (with no HF as reference). For patients with AIS the HR function for recurrence was not statistically significant throughout the follow-up period. In the AIS cohort, the HR for recurrence-free mortality was 1.69 (1.64 to 1.74$)$. For postrecurrence death, the HR was 1.45 (1.34 to 1.57). In patients with ICH, the HR function for the recurrence outcome was not statistically significant except for the period between the 157 th and the 1158th days postdischarge. The maximum value of the HR during this period was HR $(t=387)=1.79(1.18$ to 2.73$)$. For the recurrence-free mortality outcome, the HR function was not significant for the first 89 days postdischarge. Following this period, the value of the HR function increases constantly to a maximum of $\mathrm{HR}(\mathrm{t}=4108)=2.59(2.07$ to 3.26$)$ at the end of the period. HF was not associated with post-ICH mortality: $\mathrm{HR}=1.28$ (0.99 to 1.66$)$.

The results of our sensitivity analyses with adjustments confined to cardiovascular comorbidities and cancer yielded similar results (online supplementary figure 4). HF was not associated with AIS recurrence. The HR for post-AIS death was higher, HR=2.01 (1.95 to 2.07). The association between HF and ICH recurrence was significant between the 31 st and 1130th days after discharge, reaching a maximum of HR $(t=360)=1.84$ (1.22 to 2.77). The association between HF and post-ICH death was significant throughout the follow-up period: the HR function starts from a value of HR $(t=7)=1.31$ (1.03 to 1.67$)$ immediately after discharge and increases constantly until it reaches a plateau at around 1.5 years after discharge, HR $(t=449)=2.43$ (2.06 to 2.86). The function then increases constantly until the end of the follow-up period, HR $(t=4108)=2.98(2.37$ to 3.75$)$.

\section{DISCUSSION}

Our findings indicate that HF predicts worse mortality outcomes in-hospital and during the long-term follow-up, both before and after stroke recurrence. However, certain important differences between patients with AIS and patients with ICH exist. In $\mathrm{ICH}$, $\mathrm{HF}$ was not associated with an increased risk of postdischarge death in the very short follow-up (before $\sim 3$ months). After this period, the increased risk of death associated with an HF diagnosis was higher for patients with ICH than with AIS. Comparing the recurrence-free survival probabilities of patients with $\mathrm{ICH}$ with and without HF, it becomes apparent that these results may 
Table 2 Admission parameters, in-hospital complications and procedures

\begin{tabular}{|c|c|c|c|c|c|c|c|c|}
\hline & \multicolumn{2}{|l|}{ All strokes } & \multicolumn{2}{|c|}{ Acute ischaemic stroke } & \multicolumn{2}{|c|}{ Intracerebral haemorrhage } & \multicolumn{2}{|c|}{$\begin{array}{l}\text { Stroke of undetermined } \\
\text { pathology }\end{array}$} \\
\hline & HF ( $n=15397)$ & $\begin{array}{l}\text { No HF }(n=593 \\
\text { 493) }\end{array}$ & HF $(n=11522)$ & $\begin{array}{l}\text { No HF }(n=359 \\
005)\end{array}$ & HF $(n=2464)$ & $\begin{array}{l}\text { No HF }(n=170 \\
772)\end{array}$ & HF $(n=1411)$ & $\begin{array}{l}\text { No } H F(n=63 \\
716)\end{array}$ \\
\hline \multicolumn{9}{|l|}{ Admission parameters } \\
\hline Fever & $147(0.95)$ & $4214(0.71)$ & $102(0.89)^{*}$ & $2646(0.74)^{*}$ & $27(1.10)^{*}$ & $1121(0.66)^{*}$ & $18(1.28)^{*}$ & $447(0.70)^{*}$ \\
\hline Hyperglycaemia & $231(1.50)$ & $7328(1.23)$ & $179(1.55)^{*}$ & $4936(1.37)^{*}$ & $30(1.22)^{*}$ & $1834(1.07)^{*}$ & $22(1.56)^{*}$ & $558(0.88)^{*}$ \\
\hline Hypoglycaemia & $360(2.34)$ & $6958(1.17)$ & $291(2.53)$ & $4683(1.30)$ & $39(1.58)$ & $1544(0.90)$ & $30(2.13)$ & 731 (1.15) \\
\hline \multicolumn{9}{|l|}{ In-hospital complications } \\
\hline Death & 3766 (24.46) & $84645(14.26)$ & $2602(22.58)$ & $28357(7.90)$ & $989(40.14)$ & $52850(30.95)$ & $175(12.40)$ & $3438(5.40)$ \\
\hline Recurrent stroke & $26(0.17)$ & $1238(0.21)$ & $17(0.15)$ & $696(0.19)$ & $6(0.24)$ & $378(0.22)$ & $3(0.21)$ & $164(0.21)$ \\
\hline Respiratory failure & 3041 (19.75) & 53090 (8.95) & $2305(20.01)$ & $21440(5.97)$ & 595 (24.15) & 29977 (17.55) & 141 (9.99) & $1673(2.63)$ \\
\hline Pneumonia & $3088(20.06)$ & $43936(7.40)$ & $2281(19.80)$ & $21845(6.08)$ & $613(24.88)$ & $19620(11.49)$ & 194 (13.75) & $2471(3.88)$ \\
\hline Sepsis & $1161(7.54)$ & $17061(2.87)$ & $881(7.65)$ & 9996 (2.78) & $195(7.91)$ & $5878(3.44)$ & $85(6.02)$ & $1187(1.86)$ \\
\hline Shock & $429(2.79)$ & $4537(0.76)$ & $347(3.01)$ & $1964(0.55)$ & $57(2.31)$ & 2309 (1.35) & $25(1.77)$ & $264(0.41)$ \\
\hline Myocardial infarction & $777(5.05)$ & $2139(0.36)$ & $619(5.37)$ & $1600(0.45)$ & $101(4.10)$ & $389(0.23)$ & $57(4.04)$ & $150(0.24)$ \\
\hline Acute kidney injury & $1790(11.63)$ & $18717(3.15)$ & $1408(12.22)$ & $12587(3.51)$ & $268(10.88)$ & $4881(2.86)$ & $114(8.08)$ & 1249 (1.96) \\
\hline Coma & $101(0.66)$ & $2532(0.43)$ & $67(0.58)$ & $1194(0.33)$ & $23(0.93)^{*}$ & $1114(0.65)^{*}$ & $11(0.78)^{*}$ & $224(0.35)^{*}$ \\
\hline Pulmonary oedema & $184(1.20)$ & $644(0.11)$ & $121(1.05)$ & $341(0.09)$ & $48(1.95)$ & $263(0.15)$ & $15(1.06)$ & $40(0.06)$ \\
\hline \multicolumn{9}{|l|}{ In-hospital procedures } \\
\hline Mechanical ventilation & $4912(31.90)$ & 114710 (19.33) & $3412(29.61)$ & $42179(11.75)$ & $1274(51.70)$ & 68272 (39.98) & $226(16.02)$ & $4259(6.68)$ \\
\hline Tracheostomy & $858(5.57)$ & $20028(3.37)$ & $488(4.24)$ & $5364(1.49)$ & $336(13.64)$ & $14151(8.29)$ & $34(2.41)$ & $513(0.81)$ \\
\hline Gastrostomy & $3(0.02)^{*}$ & $89(0.01)^{*}$ & $3(0.03)^{*}$ & $44(0.01)^{*}$ & $0(0.00)^{*}$ & $38(0.02)^{*}$ & $0(0.00)^{*}$ & $7(0.01)^{*}$ \\
\hline Thrombolysis & $302(2.62)$ & $5776(1.61)$ & $302(2.62)$ & $5776(1.61)$ & NA & NA & NA & NA \\
\hline Echocardiography & $1619(10.52)$ & $14217(2.40)$ & $1426(12.38)$ & $12008(3.34)$ & $115(4.67)$ & $1427(0.84)$ & $78(5.53)$ & $782(1.23)$ \\
\hline
\end{tabular}

All variables are presented as frequency (percentage). All between-group differences are statistically significant $(p<0.01)$ unless otherwise specified.

${ }^{*}$ Between-group difference not statistically significant $(p>0.01)$.

$H F$, heart failure; NA, not applicable.

arise from the high mortality of patients with $\mathrm{ICH}$ in the shortterm postdischarge period. Our analysis suggests that HF seems to influence outcomes only after patients with ICH stabilise and their mortality rate decreases. It has been well recognised that intracranial haemorrhagic events can cause serious cardiac side effects, ${ }^{14}$ including cardiac failure. It may thus be that patients with ICH with comorbid HF may be more likely to suffer cardiac decompensation, leading to an increased risk of death.

Our statistical models yielded no association between HF and AIS recurrence. HF was associated with recurrent $\mathrm{ICH}$ only within the first 3 years. This revealed that there may be a 'window' within the first 3 years postdischarge when patients with HF with ICH have an increased risk of both recurrent stroke and death, after which only the mortality risk persists.

It is possible that the observed changes in the significance of ICH recurrence, occurring at around 3 years postdischarge, may be attributable to the contribution of more severe stroke types.

Table 3 Influence of pre-existing heart failure on stroke mortality in hospital

\begin{tabular}{lll}
\hline & \multicolumn{2}{l}{ Death in hospital } \\
\cline { 2 - 3 } & OR $(99 \% \mathrm{Cl})$ & P value \\
\hline All strokes & $1.74(1.65$ to 1.83$)$ & $<0.001$ \\
Acute ischaemic stroke & $2.26(2.12$ to 2.41$)$ & $<0.001$ \\
Intracerebral haemorrhage & $1.39(1.24$ to 1.55$)$ & $<0.001$ \\
Stroke of undetermined pathology & $1.75(1.40$ to 2.20$)$ & $<0.001$ \\
\hline
\end{tabular}

Results in bold are statistically significant $(\mathrm{p}<0.01)$

Results of multivariable logistic regression with no heart failure as reference category Adjusted for age, sex, comorbidities (hypertension, diabetes mellitus, dyslipidaemia, ischaemic heart disease, valvular disease, atrial fibrillation, other arrhythmias, peripheral vascular disease, cancers, chronic obstructive pulmonary disease, liver disease, anaemia, alcohol-related disorders and neurodegenerative disorders) and admission parameters (fever, hypoglycaemia and hyperglycaemia)
As the patients with a more severe clinical presentation leave the cohort (as they die) at $\sim 3$ years postdischarge, it is likely that this effect diminishes and thus the HR becomes insignificant. Nevertheless, in the absence of an objective measure of stroke severity, our hypothesis cannot be tested. Future work focusing on the impact of HF on stroke outcomes should therefore account for both stroke and HF severity.

The relationship between HF and post-AIS mortality has been previously studied, ${ }^{14-8}$ 14-20 showing that the coexistent HF increases the mortality risk both in-hospital ${ }^{5-815}$ and after discharge. ${ }^{4-8} 16-20$

A previous meta-analysis ${ }^{1}$ suggests that there is a twofold increase in the risk of AIS recurrence associated with comorbid HF. Nevertheless, our results appear to show that patients with HF were not at a higher risk of recurrent AIS than those without. While this is based on observational data, our results appear to point into a similar direction as the recent COMMANDER (A Study to Assess the Effectiveness and Safety of Rivaroxaban in Reducing the Risk of Death, Myocardial Infarction, or Stroke in Participants with Heart Failure and Coronary Artery Disease Following an Episode of Decompensated Heart Failure) HF trial, which showed that rivaroxaban anticoagulation was not associated with a reduction in the rate of incident stroke in patients with $\mathrm{HF}^{21}$

Data regarding the influence of comorbid HF on post-ICH mortality are scarce. ${ }^{22}$ We have shown that the impact of HF on ICH mortality is substantial, with a twofold mortality risk increase. Since previous studies on ICH recurrence ${ }^{2324}$ have only focused on the radiological characteristics of the primary event, we are the first to evaluate the influence of comorbid HF on ICH recurrence in the long term.

The mechanisms behind the association between HF and ICH recurrence remain nevertheless unclear. It may simply be 
Acute Ischaemic Stroke

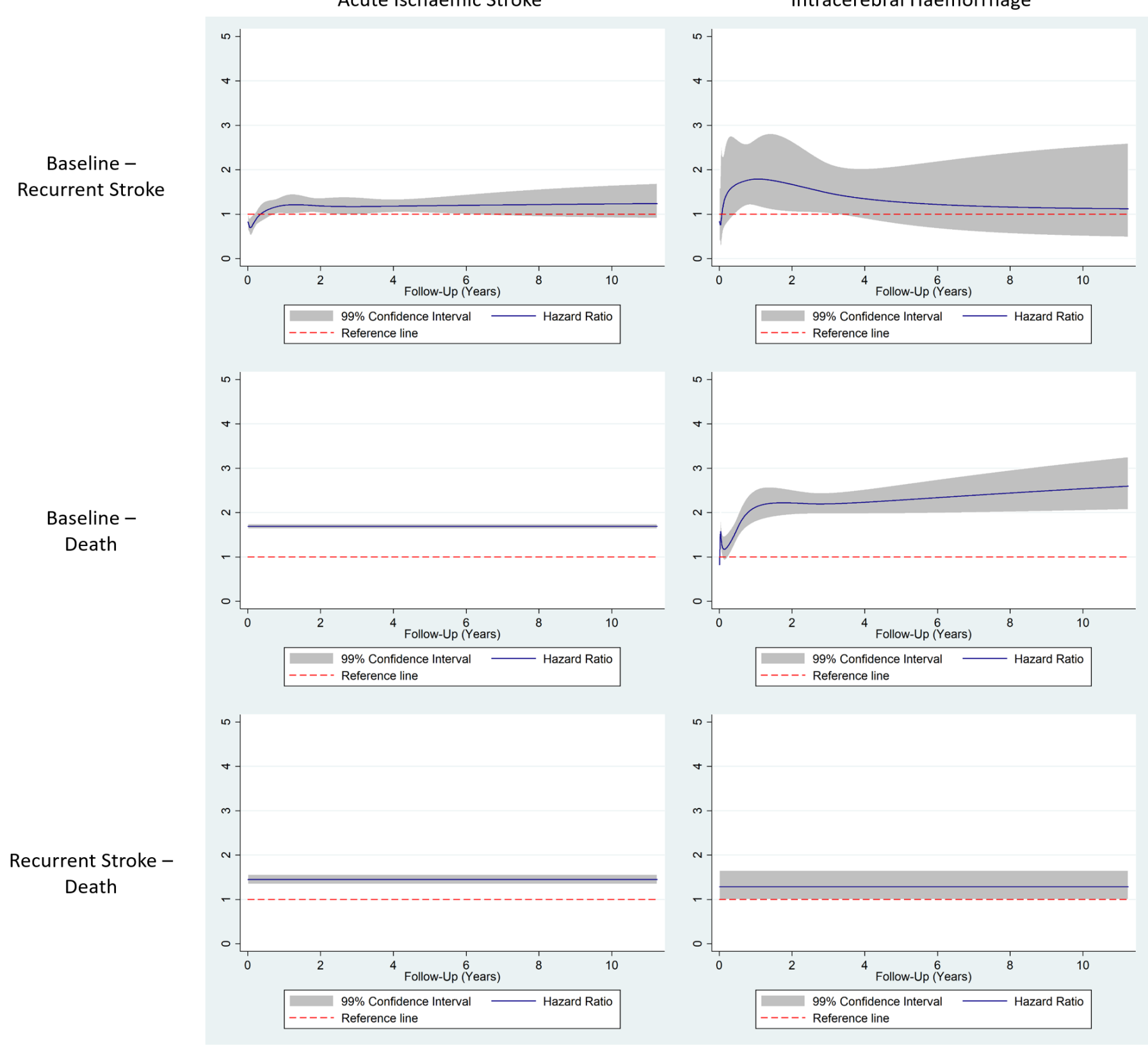

Figure 3 HR functions plotted against the postdischarge follow-up time. The solid line represents the point estimate, while the shaded area is bound by the limits of the $99 \% \mathrm{Cl}$. The dashed line represents the reference line (HR=1).

that patients with HF are more likely to receive antithrombotic therapy ${ }^{2}$ due to underlying comorbidities (coronary artery disease, valvular disease and atrial fibrillation), thus rendering these patients more prone to haemorrhagic events. ${ }^{25}{ }^{26}$ While we were unable to explore this further in the current analysis, our results suggest that caution should be exerted with HF management strategies that may cause haematological disturbances in patients with ICH. A balanced risk-versus-benefit approach may thus be desirable in the first 3 years after the acute event.

Our study has several strengths. We have used a prospectively identified, multicentre national cohort, thus minimising the potential selection bias. To the best of our knowledge, this is the first study to differentially analyse the relationship between $\mathrm{HF}$ and long-term outcomes of AIS and ICH. Our analysis provides granular insight regarding the dynamic nature of the HF-associated increased risk of death and event recurrence. This has so far not been possible in previous studies. By employing multistate survival methods and modelling the transition probabilities using flexible parametric techniques, we have managed to overcome the limitations of classical survival analysis models. ${ }^{27}$ By updating any postdischarge HF diagnoses in our statistical models, we have more accurately captured the real distribution of this comorbidity in our population. Furthermore, we have updated any incident comorbidities of interest in our all our models in the multistate model. We have for the first time illustrated the time-varying nature of the excess mortality and recurrence risk associated with comorbid HF in both stroke types. We are the first study assessing the relationship between HF and cerebrovascular disease in Asians, a population in which stroke epidemiology is markedly different. ${ }^{28} 29$

We acknowledge certain limitations. Having based all of our analyses on insurance data, case ascertainment was performed using ICD-10 codes. We thus lack data regarding stroke severity and the clinical characteristics of HF (severity, ejection fraction, 
aetiology). Given that stroke severity may be an important determinant of poststroke prognosis, it may not be possible to confirm a direct relationship between HF and adverse stroke outcomes. Furthermore, our data may not be applicable to other populations and different healthcare systems. It is important to underline that our cohort was significantly younger than Western stroke cohorts. ${ }^{5-8}$ Nevertheless, the pathophysiological link between HF and stroke is unlikely to be significantly different, highlighted by the fact that our results are concordant with previous literature. Our cohort enrolment occurred over 12 years, and time-dependent differences may exist in case ascertainment and management. Nevertheless, this remains one of the inherent limitations of long-term follow-up studies. Our data did not include any information regarding postdischarge medications or ancillary procedures, and we were thus unable to explore the effect of HF therapy or antithrombotic medication on outcomes. Finally, we were not able to evaluate the causes of death.

In summary, we report for the first time the impact of comorbid HF on the clinical trajectories of patients with acute stroke, providing stroke type-specific prognostic information. We have shown that comorbid HF significantly increases the risk of in-hospital and postdischarge death in both AIS and ICH, with the effect on patients with ICH being more pronounced. While HF was not associated with AIS recurrence, it was associated with ICH recurrence in those surviving beyond 5 months (twofold increase in relative risk) within the first 38 months. The cautious use of antithrombotic agents may be advised in patients with HF with ICH who remain at a high risk of recurrent events within the first 3 years of the acute haemorrhagic event.

Contributors PKM and TAP conceived the study. ST and KS obtained the data and ethical approval. TAP reviewed the literature and carried out statistical analysis under the supervision of ADW and ABC. TAP drafted the manuscript, and all authors contributed to the interpretation of the results and in making an important intellectual contribution to the manuscript. PKM is the guarantor.

Funding TAP received the Gwyn Seymour Aberdeen Summer Research Scholarship (ASRS) to carry out the research. ADW was funded by the Special Educational Scholarship award from the Department of Medicine for the Elderly, NHS Grampian.

Competing interests None declared.

Patient consent for publication Not required.

Ethics approval Our study protocol conforms to the ethical guidelines of the 1975 Declaration of Helsinki. Ethical approval was obtained from the Ethics Committee in Human Research, Khon Kaen University, Khon Kaen, Thailand.

Provenance and peer review Not commissioned; externally peer reviewed.

\section{REFERENCES}

1. Katsanos AH, Parissis J, Frogoudaki A, et al. Heart failure and the risk of ischemic stroke recurrence: a systematic review and meta-analysis. J Neurol SCi 2016;362:182-7.

2. Gurbel PA, Tantry US. Antiplatelet and anticoagulant agents in heart failure. JACC Heart Fail 2014;2:1-14.
3. Cuadrado-Godia E, Ois A, Roquer J. Heart failure in acute ischemic stroke. Curr Cardiol Rev 2010;6:202-13.

4. Sharma JC, Fletcher S, Vassallo M, et al. Cardiovascular disease and outcome of acute stroke: influence of pre-existing cardiac failure. Eur I Heart Fail 2000;2:145-50.

5. Divani $A A$, Vazquez $G$, Asadollahi $M$, et al. Nationwide frequency and association of heart failure on stroke outcomes in the United States. J Card Fail 2009;15:11-16.

6. Vemmos K, Ntaios G, Savvari P, et al. Stroke aetiology and predictors of outcome in patients with heart failure and acute stroke: a 10-year follow-up study. Eur J Heart Fail 2012;14:211-8.

7. Milionis $\mathrm{H}$, Faouzi $\mathrm{M}$, Cordier $\mathrm{M}$, et al. Characteristics and early and long-term outcome in patients with acute ischemic stroke and low ejection fraction. Int I Cardiol 2013;168:1082-7.

8. Pongmoragot J, Lee DS, Park TH, et al. Stroke and heart failure: clinical features, access to care, and outcomes. J Stroke Cerebrovasc Dis 2016;25:1048-56.

9. Wood AD, Mannu GS, Clark AB, et al. Rheumatic mitral valve disease is associated with worse outcomes in stroke. Stroke 2016;47:2695-701.

10. Cumming K, Tiamkao S, Kongbunkiat K, et al. Impact of HIV on inpatient mortality and complications in stroke in Thailand: a national database study. Epidemiol Infect 2017;145:1285-91.

11. Crowther MJ, Abrams KR, Lambert PC. Flexible parametric joint modelling of longitudinal and survival data. Stat Med 2012;31:4456-71.

12. Crowther MJ, Lambert PC. Parametric multistate survival models: flexible modelling allowing transition-specific distributions with application to estimating clinically useful measures of effect differences. Stat Med 2017;36:4719-42.

13. Rutherford MJ, Crowther MJ, Lambert PC. The use of restricted cubic splines to approximate complex hazard functions in the analysis of time-to-event data: a simulation study. I Stat Comput Simul 2015;85:777-93.

14. Putaala J, Lehto M, Meretoja A, et al. In-hospital cardiac complications after intracerebral hemorrhage. Int I Stroke 2014;9:741-6.

15. Appelros $P$, Nydevik I, Seiger $A$, et al. Predictors of severe stroke: influence of preexisting dementia and cardiac disorders. Stroke 2002;33:2357-62.

16. Ois $A$, Cuadrado-Godia E, Jiménez-Conde J, et al. Early arterial study in the prediction of mortality after acute ischemic stroke. Stroke 2007;38:2085-9.

17. Ois A, Gomis M, Cuadrado-Godia E, et al. Heart failure in acute ischemic stroke. $J$ Neurol 2008;255:385-9.

18. Kim W-J, Nah H-W, Kim D-H, et al. Association between left ventricular dysfunction and functional outcomes at three months in acute ischemic stroke. J Stroke Cerebrovasc Dis 2016;25:2247-52.

19. Ryu W-S, Park J-B, Ko S-B, et al. Diastolic dysfunction and outcome in acute ischemic stroke. Cerebrovasc Dis 2016;41:148-55.

20. Yousufuddin M, Bartley AC, Alsawas M, et al. Impact of multiple chronic conditions in patients hospitalized with stroke and transient ischemic attack. J Stroke Cerebrovasc Dis 2017;26:1239-48.

21. Zannad F, Anker SD, Byra WM, et al. Rivaroxaban in patients with heart failure, sinus rhythm, and coronary disease. N Eng/ J Med 2018;379:1332-42.

22. Sacco RL, Wolf PA, Kannel WB, et al. Survival and recurrence following stroke. The Framingham study. Stroke 1982;13:290-5.

23. Hill $M D$, Silver $F L$, Austin $P C$, et al. Rate of stroke recurrence in patients with primary intracerebral hemorrhage. Stroke 2000;31:123-7.

24. Zia E, Engström G, Svensson PJ, et al. Three-year survival and stroke recurrence rates in patients with primary intracerebral hemorrhage. Stroke 2009;40:3567-73.

25. Loscalzo J. From clinical observation to mechanism--Heyde's syndrome. N Eng/ I Med 2012;367:1954-6.

26. Blackshear JL, Wysokinska EM, Safford RE, et al. Shear stress-associated acquired von Willebrand syndrome in patients with mitral regurgitation. J Thromb Haemost 2014;12:1966-74.

27. Royston P, Parmar MKB. Flexible parametric proportional-hazards and proportionalodds models for censored survival data, with application to prognostic modelling and estimation of treatment effects. Stat Med 2002;21:2175-97.

28. Burke TA, Venketasubramanian RN. The epidemiology of stroke in the East Asian region: a literature-based review. Int I Stroke 2006;1:208-15.

29. Mehndiratta MM, Khan M, Mehndiratta $P$, et al. Stroke in Asia: geographical variations and temporal trends. J Neurol Neurosurg Psychiatry 2014;85:1308-12. 